\title{
Latent capacities for gametogenic cycling in the semelparous invertebrate Nereis
}

\author{
(endocrinology of reproduction/reproductive strategies/control of senescence/evolution of life histories)
}

\author{
David W. Golding and Edy Yuwono
}

Department of Marine Sciences and Coastal Management, and Dove Marine Laboratory, University of Newcastle upon Tyne, NE1 7RU, United Kingdom

Communicated by Howard A. Bern, August 4, 1994

\begin{abstract}
Most nereid polychaetes are strictly semelparous, a single episode of reproduction being invariably followed by death. Endocrine manipulation in Nereis diversicolor by the regular implantation of cerebral ganglia from immature donors unveils characteristics associated with a capacity to engage in repeated gametogenic cycling. Such manipulation permits full maturation of the gametes but blocks spawning. Gamete resorption then leads on to another bout of gametogenesis and a new cohort of gametes is formed. The neurosecretory system adopts a cyclical pattern of activity, which parallels that of gametogenesis. Repair and maintenance of the soma continue throughout sexual maturation, as shown by the persistence of feeding and the capacity for regenerative segment proliferation. In consequence, life is extended apparently indefinitely. These latent capacities are reminiscent of features of iteroparous life histories, characterized by repeated breeding, and are postulated to be vestiges of an iteroparous ancestry. They also constitute a preadaptation for iteroparity and reveal how readily a reversal to this condition could occur. The study suggests that reproductive strategies may be unexpectedly labile in even their most fundamental aspects.
\end{abstract}

Two fundamentally distinct types of reproductive strategy are encountered among multicellular organisms, involving a single breeding episode or repeated episodes. A semelparous (1) [monotelic (2) or, in plants, monocarpic (3)] strategy involves an extreme form of "reproductive recklessness" (4), with resources being diverted from the soma for investment in reproduction on a scale that apparently precludes the possibility of survival by the parent [see review by Stearns (5)]. Semelparous organisms provide valuable models for the study of senescence because of the rapid and highly deterministic character of the process in these organisms. In contrast, an iteroparous (polytelic; polycarpic) life history involves repeated bouts of reproductive activity during which repair and maintenance of the soma are maintained; the progress of senescence and the time of death are not precisely "programmed."

Most nereid polychaetes are strictly semelparous. Stages of larval and juvenile development are followed by a prolonged period of gametogonial proliferation resulting, in females, in the presence of oocytes at diverse stages of development (6). The final stages of oogenesis involve a process of "cohort formation," during which small oocytes disappear and all the gametes attain their full size, leading by a process of cytoplasmic transformation to the production of mature, fertile oocytes. The final stages of spermatogenesis involve the initiation of meiosis and the breakup of spermatocyte clusters to form motile spermatozoa. Mature nereids cease feeding and lack the capacity to regenerate caudal segments (7). In both sexes, gamete maturation is

The publication costs of this article were defrayed in part by page charge payment. This article must therefore be hereby marked "advertisement" in accordance with 18 U.S.C. $\$ 1734$ solely to indicate this fact. rapidly and invariably followed by spawning and death. In most species, although not in the estuarine Nereis diversicolor, sexual maturation is accompanied by somatic metamorphosis, or epitoky [see review by Clark (8)], fitting the animals to leave their burrows and take up a brief pelagic existence during which they swarm and spawn in the sea.

Growth, gametogenesis, and metamorphosis in nereids have long been known to be subject to the influence of a hormone [ref. 9; see review by Bentley and Pacey (10)] produced by the cerebral neuroendocrine system [see review by Golding (11)]. The hormone is indispensable for segment regeneration and has a complex action on gametogenesis. A gametotrophic aspect is evidenced by the dependence of young oocytes on the hormone for their normal development. An inhibitory aspect is also prominent; decerebration may provoke precocious maturation and this "l'effet Durchon" (12) can be blocked by replacement therapy. During the early stages of life, cerebral endocrine activity is high; its later reduction results, at least in part, from the accumulation of maturing gametes, an effect mediated by a chemical factor. Secretory activity is virtually extinguished prior to death (7).

The level of endocrine activity of a transplanted cerebral ganglion initially reflects the level in situ (7) and this intrinsic rate of activity is maintained for a prolonged period (some months) after implantation into a host at a different stage (13, 14). Consequently, in view of the well established inhibitory action of the brain hormone, we anticipated that the final stage of gametogenesis would be blocked indefinitely by the regular implantation of ganglia from immature donors. In contrast, exploratory investigations revealed that gamete maturation proceeded normally, with at most a brief delay (details will be reported elsewhere). However, such manipulation completely blocked spawning and, during the extended life of the specimens, resorption of the gametes was observed.

Resorption of unspawned gametes is a characteristic of the reproductive strategies of iteroparous polychaetes (15). However, its adaptive significance for $N$. diversicolor seemed difficult to understand-it is not, apparently, a feature of the life of this species in nature. We therefore decided to determine whether other capacities reminiscent of those associated with iteroparity are latent within this species.

\section{MATERIALS AND METHODS}

Specimens of $N$. diversicolor were collected from the River Blyth, Northumberland. The sex and stage of development of each animal and the condition of the gametes were determined by microscopic examination of a sample of coelomic fluid (the various stages of oogenesis that can be distinguished, including that at which the oocytes are considered to be fully mature, are specified in ref. 7). Experiments were set up in the fall and winter using large specimens due to breed the following spring (6). In the fall, such animals, if female, contain a large but heterogeneous population of oocytes; if male, the coelom contains myriads of spermatogo- 
nial clusters. Their cerebral ganglia exhibit high levels of endocrine activity (7).

Endocrine manipulation involved the intracoelomic implantation of two cerebral ganglia removed from small, immature donors (females with a maximum oocyte diameter of $<100 \mu \mathrm{m}$, due to breed at least 1 year after the hosts) into each large host at bimonthly intervals. Implanted specimens and sham-operated controls were maintained at $10^{\circ} \mathrm{C}$ unless otherwise stated and were fed weekly with a proprietary fish food.

The endocrine activity of cerebral ganglia was assayed by implantation into decerebrate, immature, female hosts from which all but the first 30 segments had been amputated. The hosts were then maintained together at $20^{\circ} \mathrm{C}$ for 3 weeks. Actively secreting ganglia exhibit gametotrophic activity, maintaining host oocytes with a normal cytology, and induce prolific segment regeneration. Inactive ganglia lack gametotrophic activity and induce little regeneration (see ref. 7).

The feeding activity of manipulated specimens was monitored by direct observation of the behavior and by noting the disappearance of the food and the appearance of fecal pellets. Regenerative ability was investigated by removal of all but the first 40 segments. After 2 months, the numbers of segments regenerated-i.e., in the first bout of regeneration-were counted. All segments regenerated, plus one old segment, were then amputated and, after a further 2 months, the numbers of segments proliferated in the second bout were counted.

\section{RESULTS}

Gametogenic Cycling. Specimens subjected to regular ganglion implantation proceed normally through the processes of cohort formation (see above) and final gamete maturation (further details will be provided elsewhere). They fail to spawn, although large numbers of gametes may be lost from the coelom during ganglion implantation and coelomic sampling. Subsequently, they invariably proceed to resorb their gametes. This process is frequently followed by initiation of a new cycle of gametogenesis (Table 1; Fig. 1), often after an asexual phase, bringing the specimens back toward the stage in the gametogenic cycle at which the experiment had started. The reappearance of young gametes in the coelom is poorly synchronized between individuals.

Life in manipulated nereids is apparently prolonged indefinitely, although attrition occurs due to mortality. associated with infection, damage inflicted by the regular implantation procedure, or development of large accumulations of degenerating gametes that may occlude the coelom.

In another experiment commenced in the fall, one group of eight females was subjected to regular ganglion implantation and maintained under near ambient conditions throughout

Table 1. Gametogenic cycling

\begin{tabular}{|c|c|c|}
\hline & \multicolumn{2}{|c|}{ Controls/implanted } \\
\hline & Females & Males \\
\hline No. of specimens initially & $38 / 38$ & $10 / 10$ \\
\hline No. surviving the experiment* & $0 / 25$ & $0 / 5$ \\
\hline No. resorbing mature gametes ${ }^{\dagger}$ & $0 / 25$ & $0 / 5$ \\
\hline No. with gametogenic reactivation $\ddagger$ & $0 / 18$ & $0 / 5$ \\
\hline
\end{tabular}

The experiment was set up several months before the breeding season and terminated when all the controls had spawned and died.

*Number surviving when the experiment was terminated.

tCumulative total of the number of survivors that showed this phenomenon-not all of those involved were still actively engaged in this process at the end of the experiment.

$\ddagger$ Number of survivors showing renewed gametogonial proliferation; other implanted specimens that did not survive until the end of the experiment also showed resorption and reactivation.
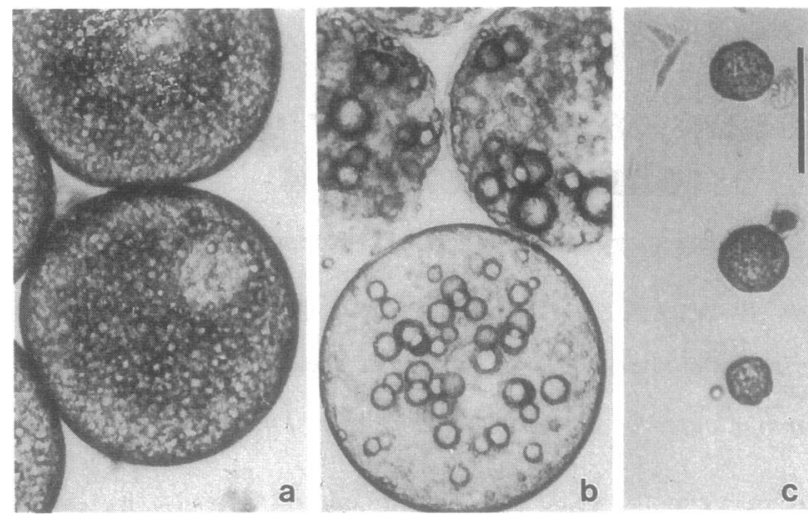

Fig. 1. Unfixed oocytes of implanted specimens. Gametogenesis proceeds normally to produce mature oocytes $(a)$; it is followed by degeneration $(b)$ and later, in many cases, by renewed gametogenesis (c). $($ Bar $=100 \mu \mathrm{m}$.)

the breeding season. Cohort formation and final maturation occurred in every case, but spawning was blocked. Three individuals survived until the following breeding season, by which time they had brought another cohort of oocytes to maturity. For the second time, spawning was blocked, life was extended, and gamete resorption was initiated. The three specimens were then discarded, 21 months after the beginning of the experiment.

A second group of eight females was initially treated identically to the first, but from June onward (when all 10 controls had spawned and died) they received no further implants. Three specimens survived until the following spring and, of these, two had developed a large number of apparently normal, mature oocytes. These two spawned normally and died. These experiments reveal a latent capacity to engage in repeated cycles of gametogenesis and to bring a cohort of gametes to maturity at the appropriate time of each year.

Endocrine Reactivation. In an experiment set up in the fall, we investigated $(i)$ whether the ganglia of manipulated specimens are inactivated during gamete maturation and (ii) whether the system is reactivated when the animals have resorbed their unspawned gametes and returned to a sexually immature condition.

Four months after the start of the experiment, coelomic sampling confirmed that all the specimens contained homogeneous populations of mature gametes. The specimens were then randomly divided into two groups and the ganglia (i.e., their own ganglia, not those implanted within them) of the first group of 10 were assayed for endocrine activity. All the ganglia (Table 2, mature) were virtually inactive as judged by their failure either to exhibit gametotrophic activity or to induce prolific segment regeneration. The ganglia of newly collected, immature animals (Table 2 , immature) were assayed at the same time for comparison; all were found to be highly active on both counts, confirming the validity of the assay procedure.

The second group of manipulated specimens $(n=20)$ were maintained for an additional 4 months-i.e., well beyond the normal period of spawning and death, during which gamete resorption occurred. The ganglia of those remaining alive (Table 2, postmature) were then assayed. A significant proportion (6 of 14) were shown to have a high level of endocrine activity; they exhibited gametotrophic activity and induced prolific regeneration. The other eight had remained inactive as judged by both criteria. A control was provided by a group of decerebrate, immature specimens that received no ganglionic implants (Table 2, none). All of these lacked any source of gametotrophic activity and regenerated few segments, 
Table 2. Endocrine reactivation

\begin{tabular}{lcccc}
\hline & \multicolumn{4}{c}{ Type of ganglia } \\
\cline { 2 - 5 } & Mature & Immature & Postmature & None \\
\hline No. of hosts* $^{*}$ & 10 & 10 & 16 & 10 \\
No. surviving & 10 & 8 & 14 & 9 \\
No. with trophic act. & 0 & 8 & 6 & 0 \\
Segments regenerated & - & 9.4 & 8.0 & - \\
SE & - & \pm 0.9 & \pm 0.7 & - \\
No. w/o trophic act. & 10 & 0 & 8 & 9 \\
Segments & & & & \\
$\quad$ regenerated & 3.3 & - & 2.5 & 1.6 \\
SE & \pm 0.5 & - & \pm 0.3 & \pm 0.3 \\
\hline
\end{tabular}

Cerebral ganglia were assayed for endocrine activity by implantation into decerebrate, immature hosts lacking posterior segments. Types of ganglia: mature, from mature donors subjected to endocrine manipulation; immature, from immature donors; postmature, from manipulated donors maintained for $\mathbf{4}$ months after maturation; none, results from decerebrate immature specimens that received no ganglion implants.

*Initial number of hosts.

tNumber of hosts surviving the assay procedure.

$\ddagger$ Number of surviving hosts containing normal oocytes, indicating that ganglia implanted within them possessed gametotrophic activity.

\$Mean number of segments regenerated by these hosts.

Number of surviving hosts containing abnormal oocytes, indicating that ganglia implanted within them (if any) lacked gametotrophic activity.

"Mean number regenerated by these hosts.

confirming that the positive results recorded above for some of the postmature ganglia were indeed due to the endocrine activity of the latter.

The results confirm that virtual extinction of cerebral endocrine activity occurs during sexual maturation (despite endocrine manipulation) and show further that restoration of activity can occur in nereids induced to resorb their gametes and return to an immature condition. Nereids possess a latent capacity for endocrine cycling.

Repair and Maintenance of the Soma During Sexual Maturation. The decline in feeding activity on the part of females approaching sexual maturation was monitored (Fig. 2) and,

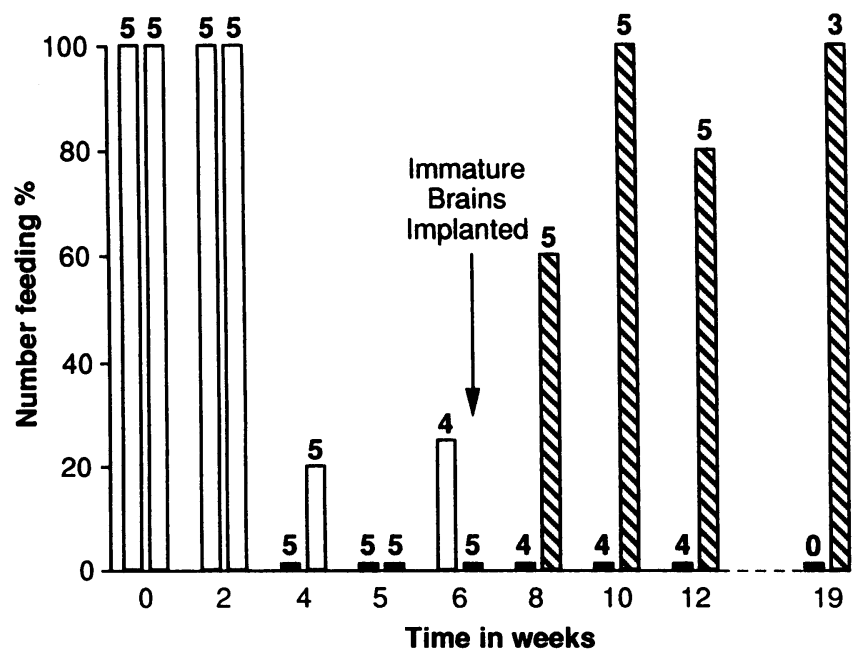

Fig. 2. Incidence of feeding was followed in two groups, each initially composed of five specimens. When feeding activity was virtually extinguished, bimonthly ganglion implantation was commenced in one group only (timing of initial implantation 6 weeks after the start of the experiment is indicated by arrow). Time in weeks, time from the start of the experiment; numbers above bars are numbers surviving at any given time; hatched bars, implanted specimens. when it had virtually ceased, immature ganglia were implanted. Feeding was resumed, whereas the behavior failed to reappear in the controls. Another experiment revealed that feeding can be resumed even after the climax of gamete maturation, when it has been extinguished for more than a month. Four of five specimens reacted positively to manipulation, and three were still feeding 4 months later, 3 months after the death of the five controls. However, feeding is irretrievable once spawning has commenced (five experimental specimens). When implantation was commenced before feeding ceased (e.g., in the endocrine reactivation experiment), this behavior continued without intermission throughout the period of cohort formation and gamete maturation.

The loss of regenerative ability during maturation can also be reversed (at least in part) by endocrine manipulation (Table 3). Whereas the ability had been extinguished in controls, no less than 15 cases were recorded, in relation to animals receiving implants of cerebral ganglia, in which the number of segments regenerated by an individual in a single bout of regeneration exceeded six. These experiments reveal a latent capacity to sustain repair and maintenance of the soma throughout gamete growth and maturation.

\section{DISCUSSION}

Significance of Senescence in Nereis. In semelparous life histories such as that of Nereis, an intimate relationship exists between reproduction on the one hand and senescence and death on the other. These phenomena are often timed with great precision. For example, the cuttlefish (Sepietta oweniana), which lays eggs with protective capsules, may die within a day after mating and spawning (16). In contrast, female Octopus cyanea survive for a month after spawning, during which time they brood their eggs (which are otherwise unprotected) and generally die a few days after the eggs hatch (17).

Semelparous reproductive strategies are usually regarded as involving an extreme form of "reproductive recklessness" (4), support for the soma being abandoned in order to concentrate resources on breeding. Thus, castration of $\mathrm{Pa}$ cific salmon (18), removal of the seeds from annual (bean) plants (3), and extirpation of the optic glands (which promote gonad development) in Octopus (19) alike prolong the lives of the parental organisms. Prevention of the frenzied sexual activity of certain male marsupial mice similarly delays death (20).

The extension of life in manipulated nereids is clearly similar to the above, but its precise basis is not clear. It should not be attributed mainly to the blockade of spawning and the reclamation by the soma of the resources invested in gametogenesis during gamete resorption, since the latter typically occurs after the normal time for spawning and death. Instead, reproductive recklessness is probably moderated-i.e., maintenance of the soma is not abandoned so completely as it is normally during maturation. The regeneration experiment provides evidence for this. Then is the ability of manipulated nereids to sustain somatic viability while bringing a large cohort of gametes to maturity inconsistent with the idea of a trade-off between somatic viability and reproductive effort? Probably not-manipulated specimens may mobilize fewer resources from the soma and produce fewer gametes with less ample stores of yolk. Further investigation is required in these areas.

Adaptive theories of senescence account for the phenomenon on the basis of the accelerated succession of generations and thus the potential for an enhanced rate of adaptive evolution $(21,22)$ or, alternatively, on the basis of the limitation of population growth (19), that result from its operation. However, such theories imply the existence of some form of altruism; they depend on the operation of group 
Table 3. Restoration of regenerative ability

\begin{tabular}{lcccccc}
\hline & \multicolumn{7}{c}{ Experiment (bout) } \\
\cline { 2 - 7 } & $1(1)$ & $1(2)$ & $2(1)$ & $2(2)$ & $3(1)$ & $3(2)$ \\
\hline No. surviving & $12 / 12$ & $4 / 9$ & $8 / 12$ & $0 / 9$ & $6 / 10$ & $0 / 8$ \\
Segments regen.* & $0 / 1.7$ & $0 / 3.1$ & $0 / 5.3$ & $-/ 6.4$ & $0 / 3.6$ & $-/ 2.6$ \\
SE & $-/ \pm 0.6$ & $-/ \pm 1.0$ & $-/ \pm 1.5$ & $-/ \pm 1.5$ & $-/ \pm 1.4$ & $-/ \pm 0.7$ \\
\hline
\end{tabular}

Three separate experiments were set up at monthly intervals, and in each experiment opportunity was given for each specimen to regenerate during two successive periods (i.e., in bouts 1 and 2). Paired numbers relate to controls and implanted specimens, respectively (i.e., controls/implanted).

${ }^{*}$ Mean number of segments regenerated.

selection, the efficacy of which is now generally discounted except under rare and highly circumscribed conditions (reviewed in refs. 23 and 24 ), which would seem to be absent from the ecology of nereids.

Hypotheses involving contributions to individual fitness are generally regarded as being of greater validity (25) and, in the context of life history theory, usually involve the idea of trade-offs between individually advantageous but mutually incompatible features (26). The neglect of somatic repair and maintenance during the breeding season in nereids may reasonably be interpreted in this way. Feeding in $N$. diversicolor typically involves extending the body from the mouth of the burrow, rendering the animal vulnerable to predation by birds. Such behavior on the part of a sexually mature nereid would, for small potential gain, place at risk the resources accumulated during the entire lifetime; consequently, the abandonment of feeding may well enhance individual reproductive potential. Similarly, somatic regeneration in a sexually mature, semelparous annelid would require investment of resources but could contribute nothing to fecundity-regenerated segments would have no opportunity to engage in gametogenesis (27).

Significance of Semelparity in Nereis. In the natural environment, $\boldsymbol{N}$. diversicolor is semelparous. Once individuals in the population under study embark on the final stages of gametogenesis, the process is apparently irreversible; a homogeneous cohort of mature gametes is produced, cerebral endocrine activity is driven ever lower, and somatic support and repair are neglected (feeding ceases, regenerative ability is extinguished). The gametes are always spawned and death invariably ensues. [In other populations, resorption of gametes is said to occur (28), but whether this represents more than gamete degeneration in partially spawned animals during terminal senescence is doubtful.]

Our findings reveal that nereids have capacities, apparently never normally expressed, reminiscent of those associated with iteroparous reproductive strategies. Most crucially, specimens subjected to endocrine manipulation can engage in repeated bouts of gametogenesis. Spawning is blocked under such conditions (although large numbers of gametes may be shed from the coelom during surgical procedures) and gamete resorption occurs prior to the start of a new cycle of gametogenesis, but this precisely parallels the situation in those iteroparous polychaetes that occasionally abort spawning altogether (15). Clearly, the germinal epithelium retains the capacity to produce a new generation of gametes-a capacity that is not, apparently, normally utilized. Similarly, the endocrine system adopts a cyclical pattern of secretion, in step with the cycles of gametogenesis, which is identical to those involved in the production of maturation-inhibiting hormones in iteroparous syllid polychaetes (29) and nemertines (12). In the long-term experiment reported above, the endocrine system would have passed through two complete cycles-activity being initially high during gamete proliferation but extinguished during maturation-during successive breeding seasons. Last, manipulated specimens can sustain repair and maintenance of the soma throughout sexual maturation, as in iteroparous annelids (27).
Charnov and Schaffer (30) asserted that a semelparous strategy, which maximizes the allocation of resources to reproduction on a single occasion at the expense of the life of the parent, is of selective value when there is a low probability of adult survival to breed again. Salmon and eels, which undertake risky and strenuous journeys upstream to spawn, and semelparous marsupial mice (20) exemplify this connection. Marine nereids, which abandon their comparatively protected, benthic habitat to swarm and spawn in the sea as pelagic epitokes (8), are self-evidently in the same situation. $N$. diversicolor does not show epitoky and individuals probably spawn within their burrows (a pattern presumably linked to their estuarine habitat), but in its semelparous reproductive strategy, the species reveals its recent evolutionary history [review by Clark (8)].

Semelparity may, like asexuality, facilitate the exploitation of opportunities for rapid population growth. In contrast, iteroparity is thought to reduce the chances of extinction by a process of reproductive bet-hedging (reviewed in ref. 5, pp. 192-198). However, nereids engage in their own form of bet-hedging in that animals produced in any given season do not develop as a single cohort but diverge to breed during several successive seasons (6). Thus, although an individual breeds only once, the genes it bequeaths to its offspring are, like those it shares with its siblings, involved in reproduction during several seasons.

According to Cole (1), "one feels intuitively that natural selection should favor the perennial reproductive habit" and iteroparity is commonly regarded as the more effective breeding strategy. In contrast, we postulate that the latent capacities of Nereis for gametogenic cycling, etc., are echoes of a more distant, iteroparous ancestry-i.e., that semelparity in these animals is a secondary, specialized condition that has evolved from iteroparity, capacities associated with which remain in vestigial form. Consistent with this interpretation is the fact that the great majority of the near relatives of the Nereidae are iteroparous and, since Nautilus has an extended and probably iteroparous life history (31), the semelparity that characterizes most advanced cephalopods presumably has the same status. In $N$. diversicolor at least, the greater part of the genetic program for postreproductive survival may not have been lost (see ref. 26). It is therefore interesting to speculate that, since the raison d'être of semelparity in nereids does not, apparently, now apply to this species, its latent capacities for gametogenic cycling may constitute a preadaptation for iteroparity and could facilitate a return to this condition.

E.Y. was supported by a scholarship provided by the Second Indonesian University Development Project (World Bank XVII).

1. Cole, L. C. (1954) Q. Rev. Biol. 29, 103-137.

2. Wynne Edwards, V. C. (1962) Animal Dispersion in Relation to Social Behaviour (Oliver \& Boyd, Edinburgh).

3. Wareing, P. F. \& Seth, A. K. (1967) Symp. Soc. Exp. Biol. 21, 543-558.

4. Calow, P. (1979) Life Cycles, An Evolutionary Approach to the 
Physiology of Reproduction, Development and Ageing (Chapman \& Hall, London).

5. Stearns, S. C. (1992) The Evolution of Life Histories (Oxford Univ. Press, Oxford, U.K.).

6. Olive, P. J. W. \& Garwood, P. R. (1981) J. Mar. Biol. Assoc. U.K. 61, 193-213.

7. Golding, D. W. (1983) Gen. Comp. Endocrinol. 52, 456-466.

8. Clark, R. B. (1961) Biol. Rev. 36, 199-236.

9. Durchon, M. (1948) C. R. Hebd. Seances Acad. Sci. 227, 157-158.

10. Bentley, M. G. \& Pacey, A. A. (1992) in Oceanography and Marine Biology, eds. Barnes, M., Ansell, A. D. \& Gibson, R. N. (UCL, London), Vol. 30, pp. 443-481.

11. Golding, D. W. (1992) in Microscopic Anatomy of Invertebrates, eds. Harrison, F. W. \& Gardiner, S. L. (Wiley, New York), Vol. 7, pp. 153-179.

12. Bierne, J. (1970) Bull. Soc. Zool. Fr. 95, 529-543.

13. Golding, D. W. (1985) Int. J. Invert. Reprod. Dev. 8, 51-59.

14. Golding, D. W. (1987) Int. J. Invert. Reprod. Dev. 12, 281-294.

15. Olive, P. J. W., Garwood, P. R. \& Bentley, M. G. (1981) Bull. Soc. Zool. Fr. 106, 263-268.

16. Bergstrom, B. \& Summers, W. C. (1983) in Cephalopod Life
Cycles, ed. Boyle, P. R. (Academic, London), Vol. 1, pp. 75-91.

17. Van Heukelem, W. F. (1983) in Cephalopod Life Cycles, ed. Boyle, P. R. (Academic, London), Vol. 1, pp. 267-276.

18. Robertson, O. H. (1961) Proc. Natl. Acad. Sci. USA 47, 609-621.

19. Wodinsky, J. (1977) Science 198, 948-951.

20. Woolley, P. A. (1966) Symp. Zool. Soc. London 15, 281-294.

21. Weismann, A. (1882), cited in Medvedev, Z. A. (1990).

22. Medvedev, Z. A. (1990) Biol. Rev. 65, 375-398.

23. Maynard Smith, J. (1976) Q. Rev. Biol. 51, 277-283.

24. Wilson, D. S. (1983) Annu. Rev. Ecol. System 14, 159-187.

25. Kirkwood, T. B. L. \& Rose, M. R. (1991) Philos. Trans. R. Soc. London B 332, 15-24.

26. Partridge, L. \& Sibly, R. (1991) Philos. Trans. R. Soc. London $B$ 332, 3-14.

27. Golding, D. W. \& Olive, P. J. W. (1978) in Comparative Endocrinology, eds. Gaillard, P. J. \& Boer, H. H. (Elsevier, Amsterdam), pp. 117-120.

28. Dales, R. P. (1950) J. Mar. Biol. Assoc. U.K. 29, 321-359.

29. Franke, H.-D. (1983) Int. J. Invert. Reprod. 6, 229-238.

30. Charnov, E. L. \& Schaffer, W. M. (1973) Am. Nat. 107, 791-793.

31. Saunders, B. W. (1984) Science 224, 990-992. 